The Astrophysical Journal, 230:428-433, 1979 June 1

(c) 1979. The American Astronomical Society. All rights reserved. Printed in U.S.A.

\title{
STRUCTURAL AND SPECTRAL SOFT X-RAY FEATURES IN THE LUPUS REGION
}

\author{
J. Davelaar, J. A. M. Bleeker, And A. J. M. Deerenberg \\ Cosmic Ray Working Group, Huygens Laboratory, Leiden, The Netherlands \\ Y. TANAKA \\ Institute for Space and Aeronautical Science, University of Tokyo, Tokyo, Japan
}

AND

S. HaYakawa AND K. Yamashita

Department of Physics, Nagoya University, Nagoya, Japan

Received 1978 August 1; accepted 1978 December 6

\begin{abstract}
The soft X-ray structure of the Lupus enhancement was investigated during a recent rocket experiment. A counting rate profile with an angular resolution of $15^{\prime}$ is presented showing significant fine structure on the subdegree scale. At least four discrete sources are resolved from the total enhancement. Correlations with the supernova of A.D. 1006 and the Lupus radio object are discussed. No other obvious radio/optical counterparts have been found for these sources, with the possible exception of the radio source MSH 14-38.

In the direction of the Lupus radio source the spectrum comprises a foreground and a source component; both have thermal spectra including line emission with temperature ranges of $2-10 \times 10^{5} \mathrm{~K}$ and $1.9-3.4 \times 10^{6} \mathrm{~K}$.

Subject headings: nebulae: supernova remnants - radio sources: general - X-rays: general
\end{abstract}

\section{INTRODUCTION}

The Lupus region was first observed by Palmieri et al. (1972), who found that it extended over about $8^{\circ}$. On various skymaps (Bunner et al. 1972; Hayakawa et al. 1975; de Korte et al. 1976) a broad region $\left(\sim 15^{\circ}\right)$ of enhanced emission, especially in the energy band $0.4-0.8 \mathrm{keV}$, is also evident. Radio observations (Milne 1971) indicate the presence of two supernova remnants-the Lupus Loop and SN 1006. The radio diameters of these sources are 4.5 and $30^{\prime} \times 22^{\prime}$, respectively. Winkler and Laird (1975) identified the latter as a hard X-ray source $\left(T=4.5 \times 10^{7} \mathrm{~K}\right)$ with a size less than $1^{\circ}$ in extent. In this experiment two cross-scans over the Lupus bright region were made at $0.31 \mathrm{~s}^{-1}$ with soft $\mathrm{X}$-ray detectors with angular resolutions of $15^{\prime}$ and $3^{\circ}$ in an attempt to resolve fine structure in the X-ray enhancement and to perform multicolor X-ray photometry.

\section{INSTRUMENTATION}

The rocket experiment was launched from Kauai aboard a Terrier-Sandhawk combination on 1976 May 29 at $09^{\mathrm{h}} 49^{\mathrm{m}} 30^{\mathrm{s}}$ UT. The maximum altitude reached was $270 \mathrm{~km}$. A short overview of the main instrument parameters is given below. For a detailed description of the payload we refer to Bleeker $e t$ al. (1978).

The instrument package consisted of (i) a onedimensional X-ray focusing collector (XFC) with an angular resolution of $15^{\prime}$ and a geometrical area of $220 \mathrm{~cm}^{2}$ covering the range $60-1000 \mathrm{eV}$ (for redundancy reasons the XFC had two multicell proportional counters in the focal plane, which were equipped with $0.5 \mu \mathrm{m}$ polypropylene windows); (ii) four large-area counters (LACs) with stacked mesh-type collimators $(3.0 \times 10: 6 \mathrm{FWHM})$, geometrical area $660 \mathrm{~cm}^{2}$, operating in the energy range $0.1-3.0 \mathrm{keV}$. On the LACs two different polypropylene window thicknesses of $1 \mu \mathrm{m}$ and $3 \mu \mathrm{m}$ were employed to provide a two-color spectral measuring technique.

Consistency throughout the flight of $1 \mu \mathrm{m}$ and $3 \mu \mathrm{m}$ data shows that the data are free from electron contamination. A Lexan-Formvar window coating was very effective against sensitivity to ultraviolet photons down to the lower threshold of $60 \mathrm{eV}$. An in-flight check on the bright UV star $\alpha$ Vir provided an upper limit for the quantum efficiency $(E>60 \mathrm{eV})$ at $2000 \AA$ of $1.8 \times 10^{-8}$. The correction for the cosmicray-induced background was assessed during the time the nose cone was still on the payload.

The aspect solution was obtained from gyro data and positions of bright UV stars, measured in a photomultiplier-type UV sensor. The accuracy of the scan path position derived in this way was 0.5 .

\section{THE LUPUS ENHANCEMENT}

Figure 1 shows counting rate profiles as a function of galactic latitude for the X-ray focusing collector and the summed output of the $1 \mu \mathrm{m}$ and $3 \mu \mathrm{m}$ LACs in the energy band $0.4-0.8 \mathrm{keV}$. The detected soft $\mathrm{X}$-ray brightness levels indicate a strong enhancement in the Lupus region. In contrast to the $0.4-0.8 \mathrm{keV}$ LAC profile, which shows no significant brightness structure in the enhancement, the XFC shows a very 


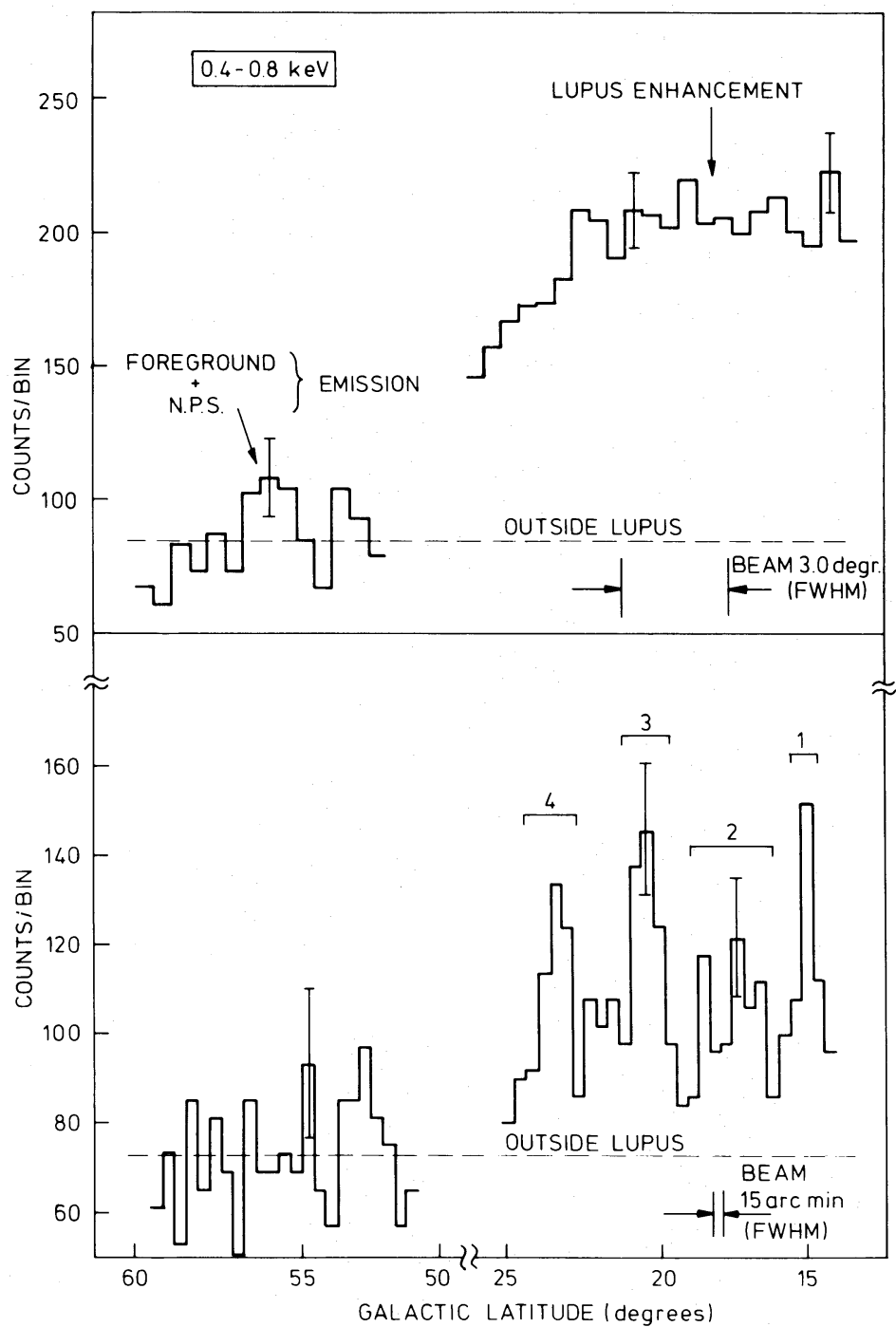

Fig. 1.-Counting rate profiles as a function of galactic latitude for the $1 \mu \mathrm{m}+3 \mu \mathrm{m}$ large-area counters (top) and the X-ray focusing collector (bottom) in the energy interval $0.4-0.8 \mathrm{keV}$. The beamwidth for each detection system is indicated.

structured counting rate profile owing to an order of magnitude improvement in the angular resolution $\left(15^{\prime}\right)$. We will now discuss the observed brightness structure in more detail.

\section{a) $S N 1006$}

Figure 2 shows the XFC counting rate profile for one scan superimposed on a radio map of Milne (1971). The source at position 1 is within 0.5 of the radio position of SN 1006. The cross-scan also shows this source to be within 0.5 of SN 1006 . In view of the 0.5 accuracy of the attitude data, indicated by the horizontal bar, it seems justified to identify this source with SN 1006 . The diameters $\left(25^{\prime}\right)$ of the X-ray and radio objects agree-a result which lends support to a supernova origin.
The spectral data (XFC) indicate a temperature in excess of $10^{7} \mathrm{~K}$ and a column density of $1.5(+3$, $-1) \times 10^{21}$ atoms cm${ }^{-2}$. These parameters are consistent with the OSO 7 result (Winkler and Laird $1975), T=(4.5 \pm 1.9) \times 10^{7} \mathrm{~K}$ and $N_{\mathrm{H}}<4 \times 10^{21}$ atoms $\mathrm{cm}^{-2}$, measured in the energy range $1-60 \mathrm{keV}$. Culhane (1976) presented a preliminary Ariel 5 spectrum in the energy range $2-18 \mathrm{keV}$. A temperature of $(1.9 \pm 0.2) \times 10^{7} \mathrm{~K}$ and a column density of $N_{\mathrm{B}}<1.4 \times 10^{22}$ atoms $\mathrm{cm}^{-2}$ are compatible with our result.

The distance, derived from the measured column density, is not incompatible with distance estimates, ranging from 1.3 to $4.9 \mathrm{kpc}$, using optical and radio observations (Minkowski 1966; Milne 1970; Downes 1971). For a spectrum with parameters $k T=4 \mathrm{keV}$ and $N_{\mathrm{H}}=1.5 \times 10^{21}$ atoms $\mathrm{cm}^{-2}$ an upper limit of 


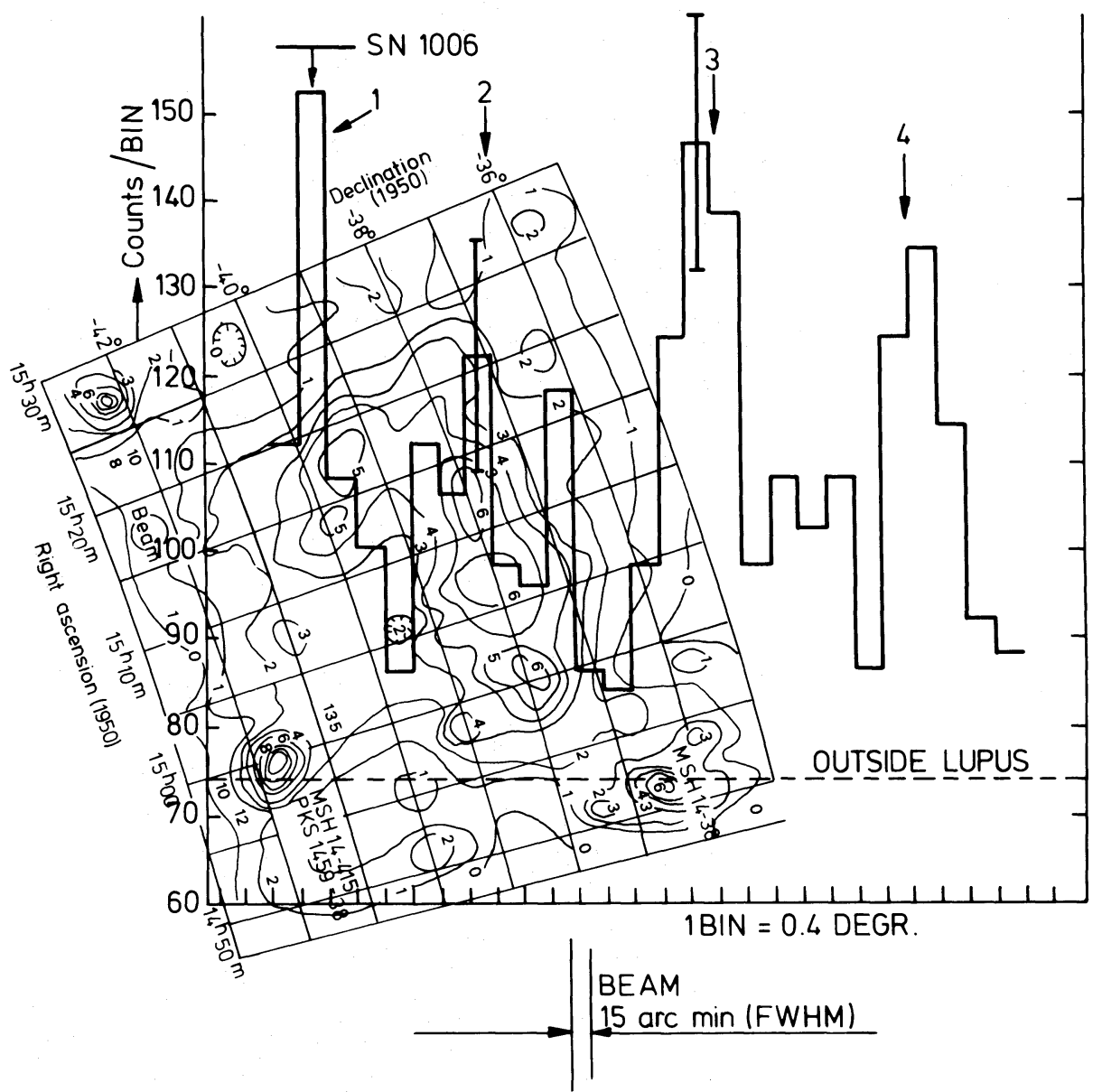

FIG. 2.-A superposition of the $0.4-0.8 \mathrm{keV}$ counting rate profile of the X-ray focusing collector on a radio map by Milne. Indicated is the measured position of $\mathrm{SN}$ 1006. The width of the bar is a composition of the source width and the uncertainty in the attitude solution.

0.09 photons $\mathrm{cm}^{-2} \mathrm{~s}^{-1} \mathrm{keV}^{-1}$ can be derived in the energy range $0.06-0.4 \mathrm{keV}$. Gronenschild (1978) obtained an upper limit of 0.05 photons $\mathrm{cm}^{-2} \mathrm{~s}^{-1} \mathrm{keV}^{-1}$ with $A N S$ in the energy range $0.16-0.28 \mathrm{keV}$. The integrated flux from 0.6 to $1.6 \mathrm{keV}$, derived from the XFC data below $1 \mathrm{keV}$, amounts to $0.24 \pm 0.07$ photons $\mathrm{cm}^{-2} \mathrm{~s}^{-1} \mathrm{keV}^{-1}$, or about a factor of 3 higher than indicated by the previous measurements. Because of this excess flux below $1 \mathrm{keV}$ we are led to conclude that SN 1006 possesses a low-temperature component similar to that found for other young remnants, Cas A and Tycho (Davison, Culhane, and Mitchell 1976), which could not have been detected by the previous experiments due to their low-energy cutoff. This low-temperature component can be interpreted in terms of the reverse shock wave model as proposed by McKee (1974) and Gull (1975). The intrinsic soft X-ray luminosity, adopting a distance of $2 \mathrm{kpc}$, amounts to $2 \times 10^{34} \mathrm{ergs} \mathrm{s}^{-1}$.

\section{b) Other Brightness Features}

In addition to SN 1006, the XFC count profile shows that the remaining portion of the Lupus en- hancement resolves into at least three sources. An extended source with a diameter of at least $3^{\circ}$ (designated 2 in Fig. 2) which, in one direction, coincides with the position of the Lupus radio source where the radio contour values are highest. Two other sources ( 3 and 4) are apparent in the counting rate profile with an angular extent of about 1:5. Because of a lower statistical significance of the XFC data in the crossscan, the spatial coincidence of source 2 with the Lupus radio source cannot be uniquely established.

Spectral analysis of the source is performed to see what additional constraints should be met for its identification. The spectral analysis is mainly based on the $1 \mu \mathrm{m}$ and $3 \mu \mathrm{m}$ LAC data during the time source 2 is visible in the XFC; the statistical weight of the XFC data does not permit placing additional constraints on the spectral parameters. Figure 3 shows the energy spectra measured in the direction of source 2 for the $1 \mu \mathrm{m}$ and $3 \mu \mathrm{m}$ LACs, respectively. Corrections have been made for cosmic-ray-induced background and the extragalactic diffuse background attenuated by the appropriate amount of interstellar gas (Daltabuit and Meyer 1972). Simultaneous fitting 

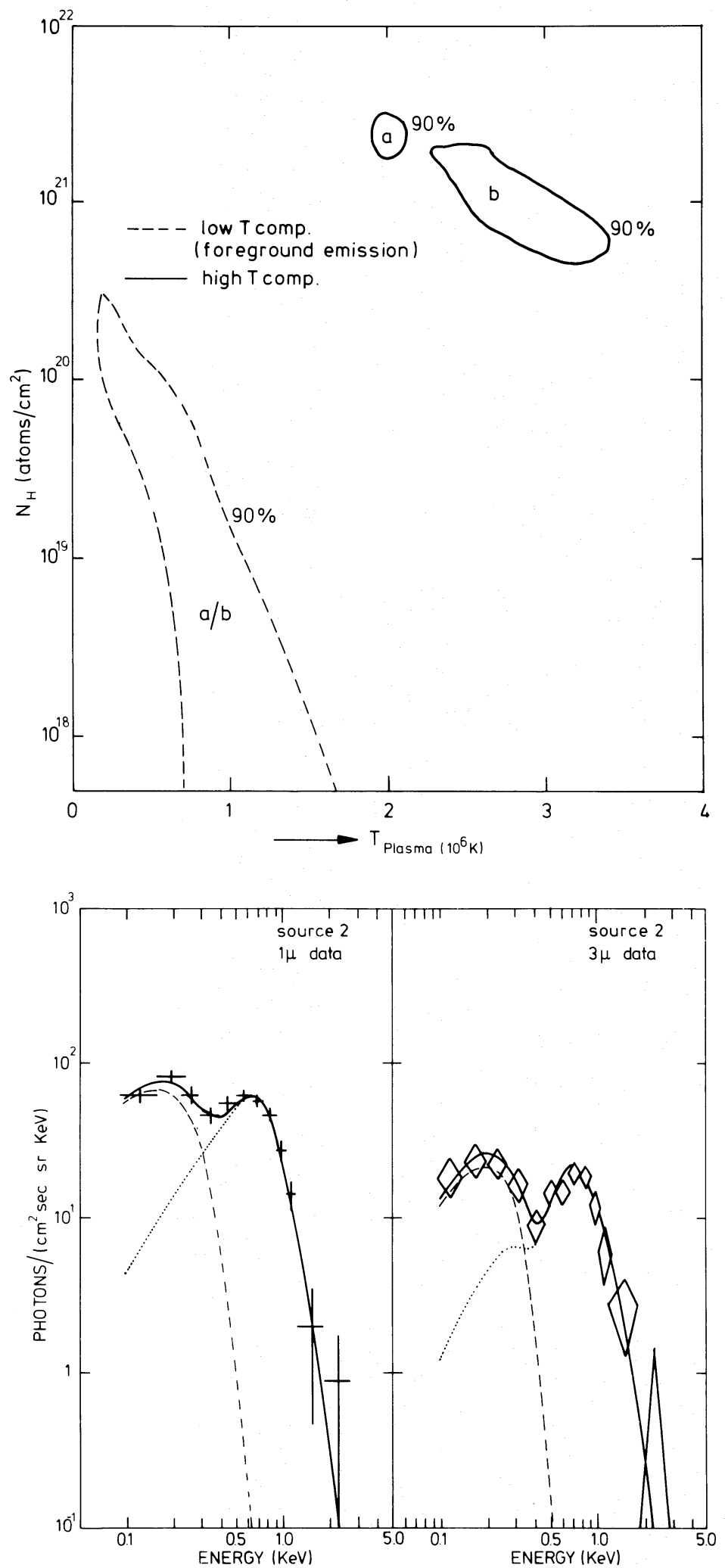

FIG. 3.-(a) (upper) Partial isoprobability contours (one component is assumed constant) indicating a $90 \%$ confidence interval for the low-temperature ("foreground") and high-temperature components. Two different abundance sets, $a$ (cosmical) and $b$ (modified), are incorporated. (b) (lower) Energy spectra of source 2 for $1 \mu \mathrm{m}$ and $3 \mu \mathrm{m}$ data. Superposed on the observational points is a combination of model spectra with modified abundances; the low $T$ component (dashed curve) for a temperature of $6 \times 10^{5} \mathrm{~K}$, the high $T$ component (dotted curve) for $3 \times 10^{6} \mathrm{~K}$. 
of $1 \mu \mathrm{m}$ and $3 \mu \mathrm{m}$ data with various model spectra (power law, free-free emission, emission from optically thin one-temperature hot plasma including line emission [Gronenschild and Mewe 1978]) yielded no acceptable fits. Therefore thin plasma spectra, comprising a low- and a high-temperature constituent, have been implemented.

Two sets of abundances have been used: $(a)$ cosmical abundances according to Mewe (1972), (b) modified abundances averaging the results obtained from the UV spectra of $\zeta$ Oph and $\lambda$ Sco (Morton 1974; York 1975). In this case the elements $\mathrm{C}, \mathrm{N}, \mathrm{Mg}, \mathrm{Si}, \mathrm{Ca}, \mathrm{Fe}$, $\mathrm{Ni}$ are depleted by factors ranging from 3 (C) to 4000 (Ca), while no depletion for $\mathrm{O}$ and $\mathrm{Ne}$ is assumed.

Both models give consistent solutions. A $\chi^{2}$ contour plot in the $\left(T, N_{\mathrm{H}}\right)$-parameter plane for both the lowtemperature and the high-temperature component is given in Figure $3 a$. Contours of $90 \%$ confidence according to the $\chi_{\min }{ }^{2}+6.25$ criterion (Lampton, Margon, and Bowyer 1976) are indicated for both abundance sets. The low-temperature contours for models $a$ and $b$ are identical, the best fit value for modified abundances is drawn superposed on the observational points for the $1 \mu \mathrm{m}$ and $3 \mu \mathrm{m}$ data in Figure $3 b$.

The low-temperature component $(T=2-10 \times$ $10^{5} \mathrm{~K}$ ) can be interpreted as "foreground" radiation, originating from a hot plasma present in the interstellar medium (Hayakawa et al. 1975; Kraushaar 1977; Tanaka and Bleeker 1977). The low column density $\left(\leq 3 \times 10^{20}\right.$ atoms $\left.\mathrm{cm}^{-2}\right)$ is consistent with the low density $\left(<0.1 \mathrm{~cm}^{-3}\right)$ within $100 \mathrm{pc}$ from the Sun, as derived from $L \alpha$ observations (Henry et al. 1976). Further support for such a hot phase of the interstellar medium is given by the $\mathrm{O}$ VI absorption line, measured in UV spectra toward six stars in the direction of Lupus (Jenkins and Meloy 1974; York 1974; Jenkins 1978). From this $\mathrm{O}$ vi abundance, which is a strong function of temperature with a maximum at $3 \times 10^{5} \mathrm{~K}$, a temperature of $6.7 \times 10^{5} \mathrm{~K}$ is determined. The temperature regime derived from the soft X-ray spectrum is consistent with this value.

The high-temperature component mainly arises from the presence of source 2 with lower limits for the amount of intervening cold gas of $N_{\mathrm{H}}=1.7 \times 10^{21}$ atoms cm-2 (cosmic abundance) or $N_{\mathrm{H}}=4.5 \times 10^{20}$ atoms $\mathrm{cm}^{-2}$ (modified abundance). A contribution up to $30 \%$ in the $0.4-0.8 \mathrm{keV}$ band to this source can be attributed to emission from the North Polar Spur (NPS), the giant soft X-ray feature in the north galactic hemisphere (Bunner et al. 1972; de Korte et al. 1974; Cruddace et al. 1976; Hayakawa et al. 1977). Spectral analysis outside the Lupus enhancement indicates a similar temperature for the NPS as for source 2 ; however, a much lower column density consistent with the $\mathrm{L} \alpha$ absorption measurements to $\alpha$ Vir ( $<10^{19}$ atoms cm-2 ; Bohlin 1975) should be inferred. It is therefore unlikely that source 2 is physically associated with the NPS. If one corrects for the contribution from the NPS, the lower limits to the $N_{\mathrm{H}}$ values of source 2 will be slightly raised. The $N_{\mathrm{H}}$ value for source 2 is compatible with the column densities for the Lupus radio source derived from other measurements. The $21 \mathrm{~cm}$ measurements toward Lupus indicate $N_{\mathrm{H}}=7 \times 10^{20}$ atoms cm $\mathrm{cm}^{-2}$ (Daltabuit and Meyer 1972). L $\alpha$ absorption measurements toward $\sigma$ Lup $(380 \mathrm{pc})$ give a mean gas density of $\eta=0.44 \mathrm{~cm}^{-3}$ beyond $100 \mathrm{pc}$ (Savage and Jenkins 1972) from which lower limits to the distance of source 2 of 340 and 1300 pc, respectively, can be deduced based on the X-ray absorption measures in models $a$ and $b$. From radio observations the distance to the Lupus Loop is estimated to be between 400 and $600 \mathrm{pc}$ (Ilovaisky and Lequeux 1972).

If we assume source 2 to be physically associated with the Lupus Loop, which is consistent with the above parameters ( $N_{\mathrm{H}}$ value, size), a supernova origin for this source is strongly favored. The spectrum shows a pronounced peak around $0.65 \mathrm{keV}$, probably indicating the presence of strong $\mathrm{O}$ VII and $\mathrm{O}$ VIII lines. Considering the temperature and angular dimension, the Lupus Loop is probably in an evolutionary sense comparable to the Cygnus Loop and Vela X. The intrinsic soft X-ray luminosity for distances of $340 \mathrm{pc}$ and $1300 \mathrm{pc}$ amounts to $1 \times 10^{34}$ ergs s${ }^{-1}$ and $2 \times 10^{35}$ ergs s$^{-1}$, respectively.

For sources 3 and 4, identified in both scans, two combinations of approximate positions can be derived $\left(\alpha=14^{\mathrm{h}} 46^{\mathrm{m}}, \delta=-33: 0\right) /\left(\alpha=14^{\mathrm{h}} 35^{\mathrm{m}}, \delta=-36: 8\right)$ and $\left(\alpha=14^{\mathrm{h}} 50^{\mathrm{m}}, \delta=-36: 0\right) /\left(\alpha=14^{\mathrm{h}} 32^{\mathrm{m}}, \delta=\right.$ -33:8). For the second combination, one position coincides with radio source MSH 14-38, a point source with a nonthermal spectrum. Taking a best fit spectral temperature of $2 \times 10^{6} \mathrm{~K}$, the $0.1-2.0 \mathrm{keV}$ $\mathrm{X}$-ray flux of this source amounts to $2.3 \times 10^{-10} \mathrm{ergs}$ $\mathrm{cm}^{-2} \mathrm{~s}^{-1}$. Alternatively the spectral similarity with source 2 might point to the picture of an extended feature in which they are somehow associated. The available radio data in the area $=-30^{\circ}$ to $-36^{\circ}$ may be found in the Ohio survey at $1415 \mathrm{MHz}$ (Ehman, Dixon, and Kraus 1970). However, no straightforward identification with any radio source could be made. No large-scale $\mathrm{H} \alpha$ nebulosities are correlated with the Lupus region (Sivan 1974).

\section{CONCLUSION}

Observing the diffuse soft X-ray background with subdegree angular resolution reveals small-scale structure. The broad Lupus enhancement is resolved into at least four separate sources. One source is identified with SN 1006, exhibiting a composite spectrum, invoking a low-temperature component. A second X-ray source of $3^{\circ}$ extent is probably associated with the Lupus Loop, indicating the existence of a supernova remnant with a temperature of about $3 \times 10^{6} \mathrm{~K}$. Considering also the linear dimension, an evolutionary resemblance to the Cygnus Loop and Vela X could exist.

The other two sources are as yet not identified with known astronomical objects, possibly apart from the radio source MSH 14-38. 


\section{REFERENCES}

Bleeker, J. A. M., et al. 1978, Astr. Ap., 69, 145.

Bohlin, R. C. 1975, Ap. J., 200, 402.

Bunner, A. N., Coleman, P. L., Kraushaar, W. L., and McCammon, D. 1972, Ap. J. (Letters), 172, L167.

Cruddace, R., Friedman, H., Fritz, G., and Shulman, S. 1976, Ap. J., 207, 888.

Culhane, J. L. 1976, Proc. Special IAU Session on Supernovae, Grenoble, 1976 September, and reference cited therein.

Daltabuit, E., and Meyer, S. 1972, Astr. Ap., 20, 415.

Davison, P. J. N., Culhane, J. L., and Mitchell, R. J. 1976, Ap. J. (Letters), 206, L37.

de Korte, P. A. J., Bleeker, J. A. M., Deerenberg, A. J. M. Hayakawa, S., Yamashita, K., and Tanaka, Y. 1976, Astr. Ap., 48, 235.

de Korte, P. A. J., Bleeker, J. A. M., Deerenberg, A. J. M., Tanaka, Y., and Yamashita, K. 1974, Ap. J. (Letters), 190, L5.

Downes, D. 1971, Ap. J., 76, 305.

Ehman, J. R., Dixon, R. S., and Kraus, J. D. 1970, A.J., 75, 351 .

Gronenschild, E. H. B. M. 1978, private communication.

Gronenschild, E. H. B. M., and Mewe, R. 1978, Solar Phys., in press.

Gull, S. F. 1975, M.N.R.A.S., 171, 263.

Hayakawa, S., Kato, T., Nagase, F., Yamashita, K., Murakami, T., and Tanaka, Y. 1977, Ap. J. (Letters), 213, L109.

Note added in proof.-The Lupus region has also been observed with the focusing collectors on $S A S 3$ (preprint submitted to $A p$.J. 1978 December). Their soft X-ray maps also reveal SN 1006 and a faint source coincident with the Lupus Loop. The "low-energy excess" of SN 1006 is also observed; however, the authors report a flux below $0.4 \mathrm{keV}$, which is inconsistent with the upper limit posed by $A N S$ and the present work.

J. A. M. Bleeker, J. DavelaAr, and A. J. M. Deerenberg: Cosmic Ray Working Group, Huygens Laboratory, Wassenaarseweg 78, Postbus 9504, 2300 RA Leiden, The Netherlands

S. Hayakawa and K. Yamashita: Department of Physics, Nagoya University, Chikusa-ku, Nagoya, Japan

Y. TANAKA: Institute for Space and Aeronautical Science, University of Tokyo, Tokyo, Japan 